\title{
Hprizons $\lambda$
}

\section{Intervenciones comunitarias y pol ít ic a gubernamental reducen el riesgo del VIH en I a República Dominicana}

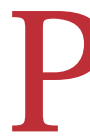

rogramas efectivos para evitar nuevas infecciones del VIH entre trabajadoras sexuales y sus parejas, y por lo tanto la población en general, son componentes críticos de estrategias nacionales de prevención del VIH .

G eneralmente esfuerzos de prevención se han concentrados en intervenciones que alcanzan a los miembros de estos grupos vulnerables al nivel individual, tal como promoción de condones y manejo de ITS. Actualmente, muchos investigadores y gerentes de programas se encuentran dirigiéndose a intervenciones "ambientales-estructurales" que toman en cuenta los contextos físicos, sociales y políticos en que el comportamiento individual occurre.

Recientemente dos tipos de intervenciones ambientales-estructurales han demonstrado aumentos en el uso de condón y reducciones en la prevalencia de ITS en las trabajadoras sexuales. U n enfoque consiste en la mobilización y desarrollo comunitario para crear un compromiso colectivo con la prevención, tal como el Proyecto Sonagachi en Calcuta, India (J ana 1998). EI segundo enfoque consiste en iniciativas patrocinadas por el gobierno, incluyendo el "Programa 100\%Condones" implementado en Tailandia que utiliza una política gubernamental que requiere el uso de condones en burdeles (Rojanapithayakorn 1996).

U n estudio reciente realizado por $\mathrm{H}$ orizons, en conjunto con dos O N Gs dominicanas-Centro de $\mathrm{O}$ rientación e I nvestigación I ntegral ( $\mathrm{CO}$ IN) y Centro de Promoción y Solidaridad H umana (CEPROSH ) - y el Programa $\mathrm{N}$ acional del Control de ITS y SIDA (DIGECITSS) buscaba evaluar el impacto de dos modelos ambientalesestructurales en disminuir el riesgo de VIH entre trabajadoras sexuales en la República $D$ ominicana y de comparar el costo-efectividad de los dos modelos. Los modelos, construidos sobre la base de años de experiencia adquirida a través de programas de educación entre pares de trabajadoras sexuales, combinan las fortalezas de iniciativas de solidaridad comunitaria y de políticas gubernamentales, e involucran a miembros de la comunidad en el desarrollo de ambos programa y política. 


\section{DESCRIPCIÓN DE LA INTERVENCIÓN}

Los modelos de intervención fueron desarrollados después de una extensa investigación formativa y consultación con líderes de pares. L os modelos fueron implementados en 68 establecimientos de sexo en dos ciudades dominicanas. En Santo D omingo, la capital del país, se implementó un modelo basado en la solidaridad comunitaria con respecto al uso $100 \%$ de condones, mientras que en Puerto Plata, una ciudad costeña mas pequena ubicada en el norte del país y con altos niveles de turismo, el modelo de solidaridad comunitaria fue combinado con la política y regulación gubernamental.

Actividades para construir la solidaridad incluyeron talleres y reuniones con trabajadoras sexuales, dueños y administradores de establecimiento, $y$ otros empleados, como porteros y disc jockeys, para fortalecer el compromiso colectivo con la prevención del VIH / SIDA, y particularmente para apoyar a las trabajadoras sexuales en el uso de condones con sus parejas. Estas reuniones también se enfocaron en explorar temas de confianza e intimidad en la negociación del uso de condones entre trabajadoras sexuales y sus parejas fijas que pagan y no pagan.

Para aumentar el compromiso colectivo con la prevención, se le recomendó a cada dueño de establecimiento de sexo montar afiches de 100\% Condones y otros materiales para aumentar la conciencia, y mantener bomboneras de vidrio llenas de condones en cada establecimiento. O tros recordatorios ambientales para apoyar el uso de condones incluyeron mensajes del disc jockey acerca de sexo seguro, mesas de información en las entradas de los establecimientos, y teatro participativo con clientes masculinos.

En Puerto Plata, se utilizaba el mismo modelo de intervención pero incluía además una política de $100 \%$ condones, patrocinada por el gobierno, que requirió el uso de condones entre trabajadoras sexuales y todos sus clientes. Los dueños fueron informados que ellos, y no las trabajadoras sexuales, eran responsables de asegurar cumplimiento con la política y las actividades del programa. En caso de faltas de cumplimiento, el gobierno iniciaba un proceso de advertencias, multas, y otras sanciones, incluyendo el cierre del establecimiento.

\section{MÉTODOS}

Los investigadores utilizaron un diseño de evaluación pre/ postexamen para evaluar los dos programas, con plazo de un año, que fueron realizados en 34 establecimientos de sexo en cada ciudad. Encuestas estructuradas y pruebas norutinarias de ITS fueron realizadas con una muestra transversal de aproximadamente 200 trabajadoras sexuales, de mínimo 18 años, que trabajaban en los establecimientos de sexo que formaban parte del estudio. Las mujeres fueron reclutadas en clínicas de salud gubernamentales en cada ciudad, pre- y post- intervención. Los datos fueron recolectados al inicio y al final del período de intervención de 12 meses.

En ambas ciudades, la edad mediana de las trabajadoras sexuales que participaron en el estudio fue de aproximadamente 25 años; el numero mediano de años de educación fueron siete. $M$ as del $75 \%$ eran solteras, con casi dos-tercios reportando tener una pareja fija. El numero mediano de salidas con clientes reportadas por las trabajadoras sexuales en la ultima semana fue dos en Puerto Plata (rango: 0-32) y uno en Santo D omingo (rango: 0-30).

Autoridades de salud, acompañadas por personal de las $\mathrm{N} \mathrm{G} \mathrm{s,} \mathrm{visitaron} \mathrm{los} \mathrm{establecimientos} \mathrm{en} \mathrm{ambas}$ ciudades mensualmente para evaluar el cumplimiento con cinco elementos claves: la presencia de afiches de $100 \%$ Condones, la disponibilidad de mínimo 100 condones en cada sitio, visibilidad y accesibilidad de condones para clientes y trabajadoras sexuales, la asistencia a chequeos mensuales de ITS de todas las trabajadoras sexuales del establecimiento, y la falta de un diagnóstico positivo para ITS entre las trabajadoras sexuales del establecimiento.

Además, se condicieron observaciónes participativas pre- y post-intervención entre una muestra al azar de 64 trabajadoras sexuales en cada ciudad, por personal masculino de $\mathrm{ON}$ G posando como clientes. Siguiendo un protocolo estricto de investigación, 
cada "cliente" seleccionó una trabajadora sexual y conversó con ella por aproximadamente 30 minutos en una mesa del establecimiento. Se le preguntó si estaba dispuesta a tener sexo sin un condón, presentándole cuatro razones, desarrolladas durante investigación formativa, por las cuales el no quería usar un condón.

\section{RESULTADOS}

El uso consistente de condones (U CC) incrementó significativamente en cada ciudad, aunque con diferentes tipos de clientes. EI U CC con clientes nuevos incrementó significativamente en las trabajadoras sexuales en Santo D omingo, de $75 \%$ a $94 \%$ ( $p$ <.001). En Puerto Plata, donde el U CC con clientes nuevos ya tenía un índice alto de 96\% el índice aumentó a 99\%. Solo en Puerto Plata incrementó significativamente el U CC con parejas fijas que pagan y no pagan, de $13 \%$ a $29 \%$ $(p=.001)$; en Santo D omingo, el U CC con parejas fijas incrementó levemente, de 15\% a 18\%. Estas cifras son particularmente importantes dado que el uso de condones con parejas fijas que pagan y no pagan fue mucho mas bajo comparado con el uso de condones con clientes nuevos, pre-intervencion. Adicionalmente, el estudio encontró que la mayoría de actos de sexo en el ultimo mes entre todas las participantes ocurrieron con parejas fijas que pagan, haciendo el aumento en Puerto Plata particularmente importante.

L a capacidad de las trabajadoras sexuales a rechazar el sexo inseguro aumentó significativamente solamente en Puerto Plata. Resultados de las observaciones participativas en Puerto Plata demuestran que el porcentaje de trabajadoras sexuales que rechazan el sexo sin condón después de oír las cuatro razones incrementó de $50 \%$ a $79 \%(p<.001)$. En Santo Domingo, el rechazo de sexo inseguro también incrementó de $64 \%$ a $72 \%$, pero la diferencia no fue estadísticamente significativa.

Indices de ITS bajaron casi $40 \%$ de pre- a post-intervención. Como demuestra la Figura 1, hubo reducciones significativas en ambas ciudades en la proporción de mujeres con resultados positivos de una or mas de tres ITS (gonorrea, tricomoniasis, y/ o clamidia), con descensos un poco mas sustantivos en Puerto Plata ( $p=.003)$, comparado a Santo D omingo $(p=.014)$. Estas reducciones generales se atribuyen mayormente a los descensos en ITS específicos: en Santo Domingo, la prevalencia de clamidia bajó significativamente de 16\%a $9 \%$ ( $p=.029)$, mientras que en Puerto Plata, la prevalencia de tricomoniasis bajo significativamente de $10 \%$ a $4 \%$ ( $p=.024)$.

EI U CC entre las trabajadoras sexuales fue asociado con niveles más altos de exposición a la intervención y cumplimiento de su establecimiento con la intervención. En análisis multivariable, el equipo de investigación encontró que la probabilidad del U CC entre las trabajadoras sexuales con todas sus parejas sexuales en el ultimo mes fue 1.84 (IC 1.07-3.17) veces más entre las que reportaron niveles altos de exposición a la intervención comparado con las que reportaron niveles bajos de exposición. EI U CC también fue 2.33 (IC 1.015.39) veces más entre las trabajadoras sexuales de los establecimientos con los niveles mas altos de cumplimiento observado a los elementos claves de la intervención.

Los establecimientos de sexo que participaron en la intervención en Puerto Plata demostraron un aumento signifitivo en el cumplimiento observado con elementos de la intervención. Aunque la exposición a la intervención reportada aumentó significativamente entre las trabajadoras sexuales en ambas ciudades, los niveles de cumplimiento observados con los cinco elementos claves de la intervención aumentaron significativamente, de un promedio de 2.6 en el primer mes, a 4.7 en el duodécimo mes $(p<.001)$, solamente en

Figura 1 La prevalencia de una o mas ITS entre las trabajadoras sexuales

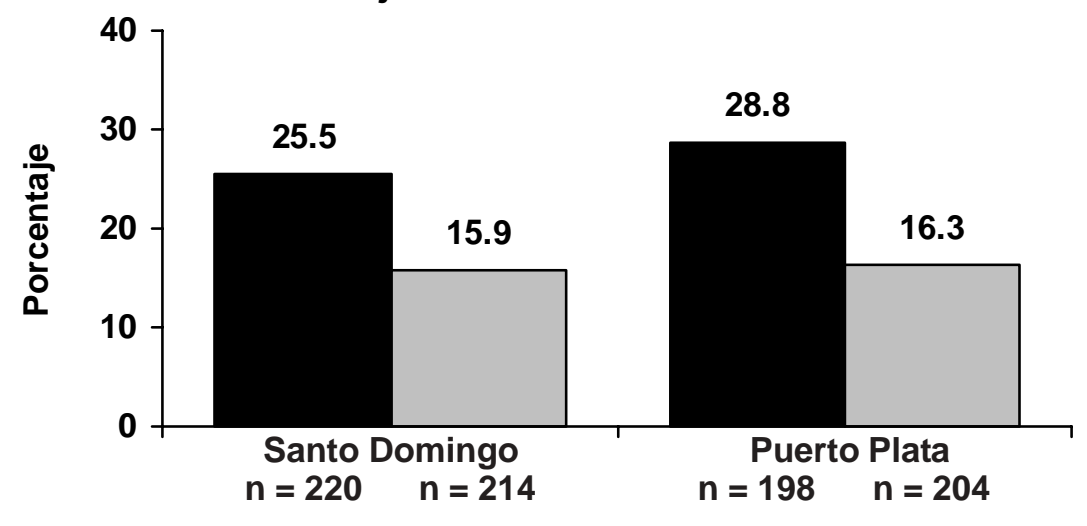

Dre-intervención $\square$ Post-intervención 
establecimientos en Puerto Plata, donde se implementó una política gubernamental requiriendo el uso de condones y un sistema graduado de sanciones para los que no lo cumplían.

\section{El modelo de Puerto Plata, que incluye regulación gubernamental, es más costo-efectivo que el modelo de Santo Domingo. LOS} investigadores hicieron un análisis de costo que demuestra que mientras el costo para implementar los dos modelos es parecido, el modelo de intervención que combinaba regulación y supervisión gubernamental es aproximadamente dos veces y medio más costo-efectivo. El costo por infección por VIH evitada en Puerto Plata, a U S\$9,843, es substantivamente menos que en Santo D omingo, a U S\$25,574. Al convertirlo, el costo por años de vida ajustados para la disabilidad (AVAD) salvado es U S\$414 en Puerto Plata, comparado con U $\$ \$ 1,075$ en Santo D omingo.

\section{CONCLUSIONES}

En ambas ciudades hubo mejoramientos de pre- a post-intervención en los variables claves de resultado del estudio: el uso consistente de condones, el rechazo del sexo inseguro, y la prevalencia de ITS. Sin embargo el tipo y el nivel de estos cambios variaron con el modelo de intervención. El modelo de Puerto Plata, que incluyó una política gubernamental y regulación, parece haber sido más exitoso en estimular aumentos significativos en el U CC con parejas fijias que pagan y que no pagan. Asimismo, el rechazo del sexo inseguro por las trabajadoras sexuales aumentó significativamente solamente en Puerto Plata.

La triangulación de estos resultados demuestra que mientras que ambos modelos tuvieron un impacto positivo en reducir la vulnerabilidad al VIH, el impacto de la intervención parece haber sido más amplio en Puerto Plata. Los datos de costoefectividad también muestran que el modelo de Puerto Plata es mucho más costo-efectivo que el modelo de Santo D omingo. Estos aumentos en Puerto Plata probablemente están vinculados a la capacidad del modelo combinado de esa ciudadsolidaridad comunitaria más política y regulación gubernamental—para alcanzar niveles mas al tos de cumplimiento con elementos claves de la intervención comparado con Santo D omingo.

$M$ ientras que existen ciertas limitaciones del estudio (p. ej. diseño pre-post, solo un modelo implementado en cada ciudad), triangulación de los datos de diferentes fuentes destacan que el modelo combinado basado en la solidaridad y la política es un paquete de intervención viable, aceptable, y efectivo para la R epública D ominicana. C on financiamiento del Fondo G lobal, el Banco M undial, y la U SAID, las O N G s implementadoras en coordinación con el gobierno dominicano están actualmente expandiendo el modelo combinado en áreas del país en donde el sexo comercial es prevalente.

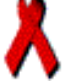

Febrero 2004

\section{REFERENCIAS}

Jana, S. et al. 1998. "ST D / H IV intervention with sex workers in West Bengal, India," AIDS 12(Suppl B): S101-8.

Rojanapithayakorn, W. and R. H anenberg. 1996. "The $100 \%$ condom program in Thailand," AID S 10(1): 1-7.

I nvestigadores para el estudio incluyen: D eanna Kerrigan, $\mathrm{H}$ orizons/ Johns H opkins U niversity; L uis M oreno y Santo Rosario, COIN; Bayardo Gomez y H ector Jerez, CEPROSH ; Ellen Weiss, H orizons/ International C enter for Research on Women (ICRW); J ohannes van Dam, H orizons/ Population Council; Eva Roca, ICRW, previamente de $\mathrm{H}$ orizons; y Clare Barrington y $\mathrm{M}$ ichael Sweat, Johns H opkins U niversity.

Para más información acerca del estudio, contacte: L uis M oreno (coin@codetel.net.do) o D eanna Kerrigan (dkerriga@jhsph.edu)

Citación sugerida: Kerrigan, D. y otros. 2004.

"I ntervenciones comunitarias y política gubernamental reducen el riesgo del VIH en la República Dominicana," Resumen de investigación de H orizons. Washington, D.C.: Population Council.

\section{Hqrizons $\lambda$}

Population Council/Horizons Communications Unit

4301 Connecticut Avenue, NW Suite 280

Washington, DC 20008

\section{Population Council}

Tel: $202-237-9400$

Fax: $202-237-8410$

horizons@pcdc.org www. popcouncil.org/horizons

Usaub Esta publicación ha sido posible gracias al apoyo brindado por la Oficina de VIH/SIDA de la Agencia Internacional para el Desarrollo de los Estados Unidos bajo los términos del Contrato HRN-A-00-97-00012-00. Las opiniones expresadas aquí son de los autores y no necesariamente reflejan los puntos de vista de la Agencia Internacional para el Desarrollo de los Estados Unidos.

๑ 2004 The Population Council, Inc.

Se puede reproducir este documento total o parcialmente sin permiso del Population Council siempre y cuando se cite la referencia completa y la reproduccion no sea con propositos comerciales. 